\title{
The IL23 axis plays a key role in the pathogenesis of IBD
}

\author{
Dermot McGovern, Fiona Powrie
}

\section{Exciting new results from a genetic study in humans and functional studies in mice have pinpointed interleukin 23 (IL23) and its receptor as a key pathway in the pathogenesis of inflammatory bowel disease (IBD). These findings reveal a hitherto unappreciated role for the IL23 axis in intestinal inflammation and may open new avenues for development of therapeutic strategies in IBD.}

See end of article for authors' affiliations

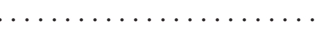

Correspondence to: Dermot McGovern, Imperial College, London, Hammersmith Hospital Campus Du Cane Road, London W12 OHS, UK; dermotmcgovern@doctors. net.uk

Revised 27 March 2007 Accepted 11 April 2007
O ver the last decade, mouse models of intestinal inflammation have provided invaluable tools to identify cytokines that drive the inflammatory response. Amongst these interleukin 12 (IL12), through its capacity to promote $\mathrm{T}$ helper type 1 (Thl) responses, emerged as a pivotal player in the development and perpetuation of colitis.

Genetic deletion or antibody-mediated neutralisation of IL12 led to amelioration of intestinal inflammation in a number of different models. ${ }^{2} 3$

However, the functional role of IL12 has been reevaluated with the discovery in 2000 of a related cytokine IL23. ${ }^{4}$ IL12 is a heterodimeric cytokine composed of a p40 and p35 subunit, whereas IL23, also a heterodimer, is composed of a unique pl9 chain linked to the p40 subunit. Many of the reagents used to assess the role of IL12 are directed against the shared IL12/IL23 p40 molecule, meaning that activities previously ascribed to IL12 may have been mediated via IL23.

The development of neutralising anti-IL23 pl9 monoclonal antibodies and IL23-deficient mice enabled investigators to distinguish between the activities of IL12 and IL23 and, in 2006, four reports identified IL23 but not IL12 as an essential mediator of intestinal inflammation. ${ }^{5-8}$ In those studies, IL23 was found to orchestrate an inflammatory cytokine cascade involving increased levels of tumour necrosis factor $\alpha$ (TNF $\alpha)$, IL6, interferon $\gamma(\operatorname{IFN} \gamma)$ and IL17 in the intestine (fig 1). Similar results were also found in models of brain ${ }^{9}$ and joint inflammation, ${ }^{10}$ suggesting that IL23 is an important conductor of the inflammatory response in tissues.

The next question is how IL23 mediates intestinal inflammation. The functional IL23 receptor (IL23R) is a heterodimer of the IL12R $\beta 1$ subunit, which is shared with the IL12 receptor and a novel IL23R subunit which is expressed by activated $T$ cells and myeloid cells. To date, attention has focused on the ability of IL23 to promote a novel subset of ILl7-producing $\mathrm{CD}^{+}{ }^{+} \mathrm{T}$ cells termed Th17 cells. ${ }^{11-13}$ These cells are distinct from Thl and Th2 cells, and recent evidence in the mouse indicates that transforming growth factor $\beta$ (TGF $\beta$ ) and IL6
Gut 2007;56:1333-1336. doi: 10.1136/gut.2006.115402

drive the differentiation of Th17 cells from naïve $\mathrm{T}$ cells. ${ }^{14}$ TGF $\beta$ and IL6 induce IL23R expression on Th17 cells, rendering them responsive to IL23. ${ }^{15}$ Accumulation of Thl7 cells is reduced in the absence of IL23, suggesting that IL23 may maintain or stabilise the Thl7 response. $^{7816}$ ILl7 is a pleiotropic cytokine that acts on both immune and non-immune cells and is increased in the intestine of IBD patients. ${ }^{17}$ IL17 can activate stromal, endothelial and epithelial cells to produce cytokines and chemokines, leading to increased neutrophil recruitment into tissues, and also induces inflammatory cytokine production by macrophages. ${ }^{18}$ Although IL17 has been shown to play an important role in IL23-driven central nervous system inflammation, ${ }^{16}{ }^{19}$ its role in intestinal inflammation is less pronounced. Neutralisation of IL17 was not sufficient to inhibit colitis in IL10deficient mice and was only partially protective when combined with anti-IL6 therapy, suggesting that in the intestine IL23 drives IL17-independent inflammatory pathways. ${ }^{5}$

Activities previously ascribed to IL12 may have been mediated via IL23

This may be explained by the recent work of Hue et $a l^{8}$ and Uhlig et $a l^{6}$ which has uncovered a novel role for IL23 in controlling innate immunity in the intestine. In these studies, $\mathrm{T}$ cell-independent colitis following intestinal bacterial infection or stimulation of the CD40 pathway was found to be dependent on IL23 and not IL12. IL23 is produced by activated myeloid cells including macrophages and dendritic cells (DCs) following bacterial stimulation $^{20}$ or via CD40 signalling, ${ }^{6}$ and drives increases in a number of inflammatory cytokines in the intestine in the absence of T cells, including TNF $\alpha$, IFN $\gamma$, IL6 and IL17. The IL23R is expressed by activated DCs and macrophages, and IL23 can induce production of inflammatory cytokines by macrophages. ${ }^{9}$ These results suggest that an important function of IL23 may be to drive an autocrine loop within the innate immune system, leading to the production of a number of inflammatory mediators that contribute to the intestinal inflammatory response.

So is there really no role for ILl2- and IFN $\gamma$ producing Thl cells in chronic intestinal inflammation? In models of autoimmune disease there is evidence that ILI2 and IFN $\gamma$ antagonise the

Abbreviations: $D C$, dendritic cell; IBD, irritable bowel disease; IFN $\gamma$, interferon $\gamma$; IL, interleukin; IL23R, interleukin 23 receptor; SNP, single-nucleotide polymorphism; TGF $\beta$, transforming growth factor $\beta$; Th, T helper; TNF $\alpha$, tumour necrosis factor $\alpha$ 


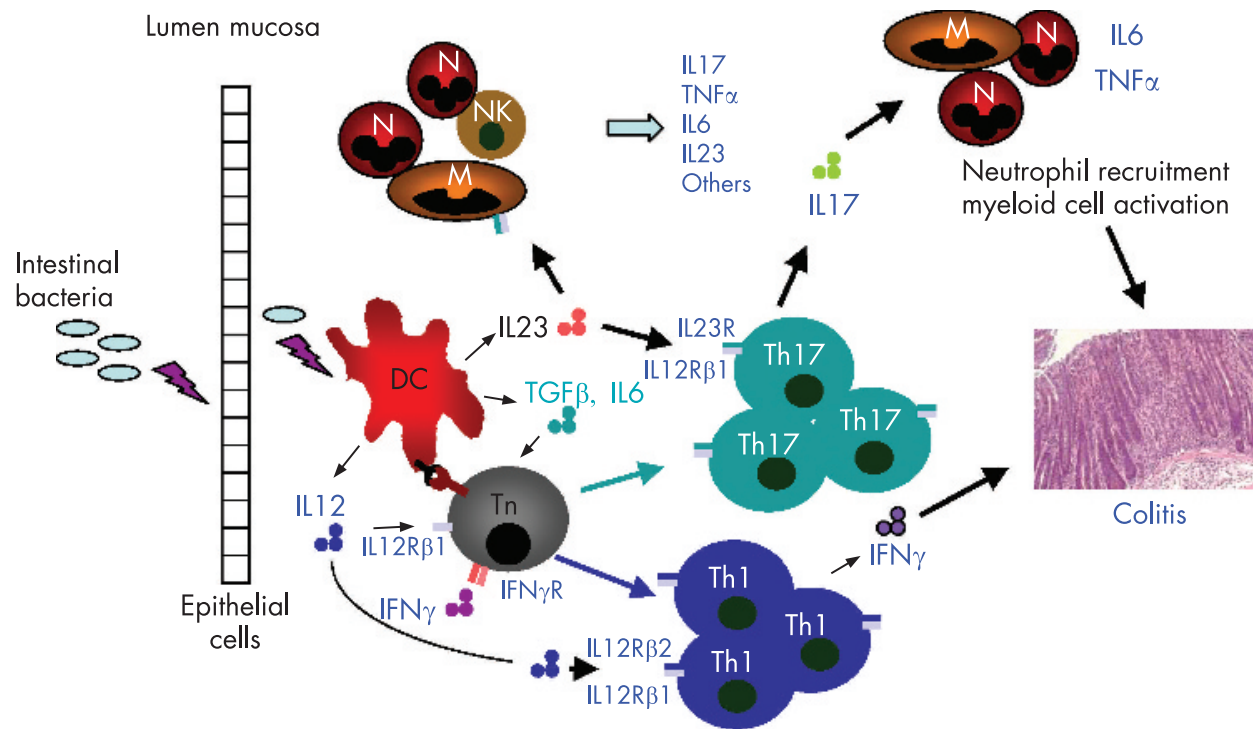

Figure 1 Interleukin 23 (IL23) orchestrates intestinal inflammation via multiple pathways. Bacterial stimulation induces cytokine production by epithelial cells, dendritic cells (DCs) and macrophages (M). Interferon $\gamma$ (IFN $\gamma$ ) and IL 12 act on antigenstimulated $\mathrm{CD} 4^{+} \mathrm{T}$ cells $(\mathrm{Tn})$ to induce the differentiation of IFN $\gamma$-secreting Thelper type 1 (Th1) cells, whereas transforming growth factor $\beta$ (TGF $\beta$ ) and IL6 promote Th 17 cells that produce IL17 and express the IL23 receptor (IL23R). IL23 produced by activated DCs sustains the Th17 response and also activates innate cells including activated myeloid cells and NK cells (NK) to produce inflammatory cytokines including IL6, tumour necrosis factor $\alpha$ (TNF $\alpha)$ and IL 17 that drive intestinal inflammation. IL17 stimulates cytokine production by activated macrophages and can also induce cytokine and chemokine production by endothelial cells, leading to neutrophil (N) recruiment. differentiation of Th17 cells. However this may be an oversimplification in the intestine as, in a model of bacteria-induced $\mathrm{T}$ cell-dependent colitis, IL23 was found to promote both IL17 and IFN $\gamma$, suggesting that these mediators interact to induce severe colitis. ${ }^{7}$ Indeed the recent studies on IL23 suggest that IL12 can contribute to intestinal inflammation, particularly in the absence of IL23, as in T cell transfer colitis a low level of intestinal inflammation remained in IL23 p19-deficient hosts but was absent in IL12 and IL23 p40-deficient mice. ${ }^{78}$ Using a model of acute intestinal inflammation, Becker et al found an increase in disease in the absence of IL23 which they attributed to an unrestrained IL12 response. ${ }^{21}$ These results serve to illustrate the complexity of immune regulatory interactions in the intestine and suggest differential roles for IL12 and IL23 in the control of acute and chronic inflammation.

\section{An important function of IL23 may be to drive an autocrine loop within the innate immune system, leading to the production of a number of inflammatory mediators that contribute to the intestinal inflammatory response.}

However, a recently published genetic study in Science has thrust the spotlight firmly on the IL23 pathway with the finding that variants of the IL23R gene are linked to IBD susceptibility. ${ }^{22}$ A North American collaborative group performed a genome-wide association scan testing over 300000 single-nucleotide polymorphisms (SNPs) across the human genome for association with Crohn's disease. This is another of what promises to be a number of genome-wide association scans to be published in IBD. In the first such study published (looking at approximately 70000 SNPs), an association with a haplotype in the TNFSF15 gene was identified in a Japanese Crohn's disease cohort, and association with both Crohn's disease and ulcerative colitis was confirmed in an independent Caucasian cohort. ${ }^{23}$ In another similar study from Germany of approximately 20000 SNPs, an association with the autophagy-related 16-like l gene (ATG16L1) gene and Crohn's disease was identified. ${ }^{24}$ These studies are powerful tools and make use of the readily available high-throughput genotyping methods now available to researchers, and have proved successful in identifying susceptibility 'genes' in other traits. ${ }^{25}$ Vast quantities of data are generated in these studies, and sorting out the true positives from the chance positive findings adds to the major problems for genetic studies investigators. In this particular study, only three (of the 300000 tested) SNPs maintained association with the risk of developing Crohn's disease after Bonferroni correction for multiple testing (at the $\mathrm{p}=0.05$ level) in their case-control study of approximately 550 Caucasian non-Jewish Crohn's cases (all with ileal disease) and approximately 550 age-, sex- and ethnically matched controls. Two of these 'positive' SNPs were in the NOD2 (CARD15) gene, a known risk factor for ileal Crohn's disease, ${ }^{26}$ a finding that provides an opportune positive 'internal' control for the study. The third 'positive' SNP was located on chromosome lp31, in a region adjacent to a known susceptibility locus for Crohn's disease, ${ }^{27}$ and encoded an amino acid change from an arginine to a glutamine residue at codon $381(\operatorname{Arg} 381 \mathrm{Gln})$ in the gene encoding a subunit of the IL23R. The authors then identified nine other markers at this locus that also showed association with Crohn's disease, thereby virtually excluding the possibility that their original finding was due to chance alone.

\section{Variants of the IL23R gene are linked to IBD susceptibility}

Importantly the authors confirmed these IL23R associations in an independent case-control cohort of Jewish ileal Crohn's disease patients and then also in a family-based association study. Rather intriguingly, an association with this IL23R variant with ulcerative colitis was also noted in the familybased association study, suggesting that the genetic susceptibility associated with $I L 23 R$ is with generalised gastrointestinal mucosal inflammation and not just with the ileal disease examined in the original cohort. These data support the theory that Crohn's disease and ulcerative colitis share some susceptibility genes but differ at other genes. The risk allele of the initially identified $I L 23 R$ variant is the common allele encoding the arginine residue and has an allele frequency of approximately 97-98\% in ileal Crohn's disease cases compared with approximately $93 \%$ in the control population. These differences in allele frequencies are small although the odds ratio and $\mathrm{p}$ value are more impressive (OR 0.26 , 95\% CI 0.15 to 0.43 , $\left.\mathrm{p}=5.1 \times 10^{-9}\right)$. A 'risk factor' that is carried by approximately 99.4\% of the 'healthy' population (the frequency of carriage of an IL23R Arg381Gln risk allele within this study's control population) is not going to be useful as a diagnostic test for Crohn's disease or IBD although, as part of a panel, as suggested elsewhere, ${ }^{28}$ it may yet prove to be more useful in the clinical setting. However, it does provide significant insight 
into disease pathogenesis and highlights potential avenues for future therapeutic intervention in IBD (see later).

\section{Mouse and human studies highlight the IL23-IL23R axis as a target for development of novel therapies for IBD.}

Two other non-synonymous (amino acid changing) IL23R SNPs were identified in this study, but showed no association with IBD. A number of IL23 intronic variants were shown to be associated with susceptibility to IBD, and the authors have speculated that these variants may occur at splicing sites and so lead to IL23R splice variants (that are known to exist) which may alter protein function. The arginine residue encoded by the 381 codon of IL23R is the fifth amino acid in the cytoplasmic domain of the receptor and is highly conserved across species, suggesting that this residue plays a crucial role in the function of the receptor. However, no 'functional' data regarding the effect of this polymorphism or the other associated variants on the normal IL23 pathway were presented in the paper. The functional studies will be eagerly awaited to see if these variants lead to a 'gain of' or 'loss of' function within this pathway-an unresolved debate that continues about the effect of the NOD2 (CARD15) variants 5 years after their identification! ${ }^{29-31}$ Replication studies by independent groups are also necessary (although the reproduction of the finding in two independent cohorts provides convincing evidence of a 'true' association) and it will also be interesting to see the results from non-Caucasian cohorts as the published data suggest that some IBD susceptibility loci are 'shared' by different ethnic groups $^{23}$ whereas others are not. ${ }^{32} 33$

\section{An IL12 p40 monoclonal antibody that neutralises IL12 and IL23 may be an effective treatment for Crohn's disease}

The genetic data complement the functional data in mice and provide a compelling case for further analysis of the IL23dependent inflammatory response in the intestine. A considerable amount of research is needed to assess the role of IL23R variants in other ethnic groups and to ascertain whether there is any epistasis (interaction) between these variants and other susceptibility genes and environmental risk factors for IBD; furthermore, large-scale studies are needed to ascertain the clinical consequences of these variants. Most pressing, however, is the need for an understanding of the functional effects of IL23R variants on the IL23 pathway.

The mouse and human studies highlight the IL23-IL23R axis as a target for development of novel therapies for IBD. Both IL12 and IL23 are increased in the intestine of patients with Crohn's disease, and there are encouraging data from early study findings that an IL12 p40 monoclonal antibody that neutralises IL12 and IL23 may be an effective treatment for Crohn's disease. ${ }^{34}$ Indeed, the best option in terms of controlling the inflammatory response may be to block the activities of both IL12 and IL23. However, IL12-dependent responses such as Thl and cytotoxic $\mathrm{T}$ cell responses play a key role in host protective immunity, and blockade of these may leave patients vulnerable to infection and cancer. The study by Uhlig et al directly addressed the roles of IL12 and IL23 in systemic and mucosal innate immunity and showed that anti-CD40-induced systemic inflammation was dependent on IL12 and not IL23, whereas the inverse was true for intestinal inflammation. These results suggest that targeting IL23 may allow a more selective block of the tissue inflammatory response while sparing systemic immunity. However, there is evidence that IL23 contributes to antibacterial immunity at mucosal surfaces, ${ }^{15} 35$ and further understanding of this pathway will be required to balance the beneficial effects of IL23 depletion on chronic inflammation with potential deleterious effects on host protective mucosal immunity.

The convergence of two widely different experimental approaches on the IL23 pathway as a key regulator in the development of intestinal inflammation is an exciting advance in IBD research. Clearly many questions remain to be answered before these results will be translated into better therapies for patients with IBD; however, this potential makes the effort worthwhile.

\section{ACKNOWLEDGEMENTS}

We thank Kevin Maloy, Philip Ahern and Jane Sutton for critical reading of the manuscript. D.M. has received support from the MRC (UK) and the IOIBD. F.P. is a Wellcome Trust Senior Research Fellow.

\section{Authors' affiliations \\ Dermot McGovern, Imperial College, London, Hammersmith Hospital Campus, Du Cane Road, London, UK \\ Fiona Powrie, Sir William Dunn School of Pathology, University of Oxford, South Parks Rd, Oxford, UK}

Competing interests: None.

\section{REFERENCES}

1 Bouma G, Strober W. The immunological and genetic basis of inflammatory bowel disease. Nat Rev Immunol 2003;3:521-33.

2 Simpson SJ, Shah S, Comiskey M, et al. T cell-mediated pathology in two models of experimental colitis depends predominantly on the interleukin 12/signal transducer and activator of transcription (Stat)- 4 pathway, but is not conditional on interferon gamma expression by T cells. J Exp Med 1998;187:1225-34.

3 Neurath MF, Fuss I, Kelsall BL, et al. Antibodies to interleukin 12 abrogate established experimental colitis in mice. J Exp Med 1995;182:1281-90.

4 Oppmann B, Lesley R, Blom B, et al. Novel p19 protein engages IL-12p40 to form a cytokine, IL-23, with biological activities similar as well as distinct from IL-12. Immunity 2000;13:715-25.

5 Yen D, Cheung J, Scheerens H, et al. IL-23 is essential for T cell-mediated colitis and promotes inflammation via IL-17 and IL-6. J Clin Invest 2006;116:1310-6.

6 Uhlig HH, McKenzie BS, Hue S, et al. Differential activity of IL-12 and IL-23 in mucosal and systemic innate immune pathology. Immunity 2006;25:309-18.

7 Kullberg MC, Jankovic D, Feng CG, et al. IL-23 plays a key role in Helicobacter hepaticus-induced T cell-dependent colitis. J Exp Med 2006;203:2485-94.

8 Hue S, Ahern P, Buonocore S, et al. Interleukin-23 drives innate and T cellmediated intestinal inflammation. J Exp Med 2006;203:2473-83.

9 Cua DJ, Sherlock J, Chen Y, et al. Interleukin-23 rather than interleukin-12 is the critical cytokine for autoimmune inflammation of the brain. Nature 2003:421:744-8.

10 Murphy CA, Langrish CL, Chen Y, et al. Divergent pro- and antiinflammatory roles for IL-23 and IL-12 in joint autoimmune inflammation. J Exp Med 2003;198:1951-7.

11 Langrish CL, Chen Y, Blumenschein WM, et al. IL-23 drives a pathogenic T cell population that induces autoimmune inflammation. J Exp Med 2005;201:233-40.

12 Aggarwal S, Ghilardi N, Xie MH, et al. Interleukin-23 promotes a distinct CD4 T cell activation state characterized by the production of interleukin-17. J Biol Chem 2003;278:1910-4.

13 Harrington LE, Hatton RD, Mangan PR, et al. Interleukin 17-producing CD4+ effector T cells develop via a lineage distinct from the Thelper type 1 and 2 lineages. Nat Immunol 2005;6(1 1):1123-32.

14 Veldhoen M, Hocking RJ, Atkins CJ, et al. TGFbeta in the context of an inflammatory cytokine milieu supports de novo differentiation of IL-17-producing T cells. Immunity 2006;24:179-89

15 Mangan PR, Harrington LE, O'Quinn DB, et al. Transforming growth factor-beta induces development of the $T(H) 17$ lineage. Nature 2006;441:231-4.

16 Chen Y, Langrish CL, McKenzie B, et al. Anti-IL-23 therapy inhibits multiple inflammatory pathways and ameliorates autoimmune encephalomyelitis. J Clin Invest 2006;116:1317-26.

17 Fujino S, Andoh A, Bamba S, et al. Increased expression of interleukin 17 in inflammatory bowel disease. Gut 2003;52:65-70.

18 Kolls JK, Linden A. Interleukin-17 family members and inflammation. Immunity 2004;21:467-76

19 Park H, Li Z, Yang XO, et al. A distinct lineage of CD4 T cells regulates tissue inflammation by producing interleukin 17. Nat Immunol 2005;6:1133-41.

20 Becker C, Wirtz S, Blessing $M$, et al. Constitutive $\mathrm{p} 40$ promoter activation and IL23 production in the terminal ileum mediated by dendritic cells. J Clin Invest 2003;112:693-706.

21 Becker C, Dornhoff H, Neufert C, et al. Cutting edge: IL-23 cross-regulates IL-12 production in T cell-dependent experimental colitis. J Immunol $2006 ; 177: 2760-4$. 
22 Duerr RH, Taylor KD, Brant SR, et al. A genome-wide association study identifies IL23R as an inflammatory bowel disease gene. Science 2006:314:1461-3.

23 Yamazaki K, McGovern D, Ragoussis J, et al. Single nucleotide polymorphisms in TNFSF15 confer susceptibility to Crohn's disease. Hum Mol Genet 2005; 14:3499-506.

24 Hampe J, Franke A, Rosenstiel P, et al. A genome-wide association scan of nonsynonymous SNPs identifies a susceptibility variant for Crohn disease in ATG16L1. Nat Genet 2007;29:207-11.

25 Li M, Atmaca-Sonmez P, Othman M, et al. CFH haplotypes without the $\mathrm{Y} 402 \mathrm{H}$ coding variant show strong association with susceptibility to age-related macular degeneration. Nat Genet 2006;38:1049-54.

26 Ahmad T, Armuzzi A, Bunce M, et al. The molecular classification of the clinical manifestations of Crohn's disease. Gastroenterology 2002;122:854-66.

27 Cho JH, Nicolae DL, Gold LH, et al. Identification of novel susceptibility loci for inflammatory bowel disease on chromosomes $1 p, 3 q$, and $4 q$ : evidence for epistasis between Ip and IBDI. Proc Natl Acad Sci USA 1998;95:7502-7.
28 McGovern DP, Butler H, Ahmad T, et al. TUCAN (CARD8) genetic variants and inflammatory bowel disease. Gastroenterology 2006;131:1190-6.

29 Kobayashi KS, Chamaillard M, Ogura Y, et al. Nod2-dependent regulation of innate and adaptive immunity in the intestinal tract. Science 2005;307:731-4.

30 Watanabe T, Kitani A, Murray PJ, et al. NOD2 is a negative regulator of Toll-like receptor 2-mediated T helper type 1 responses. Nat Immunol 2004:5:800-8.

31 Wehkamp J, Harder J, Weichenthal M, et al. NOD2 (CARD15) mutations in Crohn's disease are associated with diminished mucosal alpha-defensin expression. Gut 2004;53:1658-64.

32 Negoro K, McGovern DP, Kinouchi Y, et al. Analysis of the IBD5 locus and potential gene-gene interactions in Crohn's disease. Gut 2003;52:541-6.

33 Sugimura M, Kinouchi Y, Takahashi S, et al. CARD15/NOD2 mutational analysis in Japanese patients with Crohn's disease. Clin Genet 2003:63:160-2.

34 Mannon PJ, Fuss I, Hornung R, et al. Anti-interleukin-12 P40 antibody treats active Crohn's disease. Gut 2004;53(Suppl IV):A48.

35 Happel KI, Dubin PJ, Zheng M, et al. Divergent roles of IL-23 and IL-12 in host defense against Klebsiella pneumoniae. J Exp Med 2005;202:761-9.

\section{EDITOR'S QUIZ: GI SNAPSHOT}

\section{Multiple liver lesions in a smoker}

\section{Clinical presentation}

A 39-year-old female smoker presented to the gastrointestinal unit with a 6-month history of increasing right upper quadrant pain, abdominal distension, nausea and anorexia. There was a history of dysmenorrhoea but no bowel disturbance. There was no medical history of note.

On examination, the abdomen was distended with right upper quadrant tenderness. Investigations showed a normal full blood count and normal renal and liver biochemistry. Inflammatory and tumour markers were not elevated. An

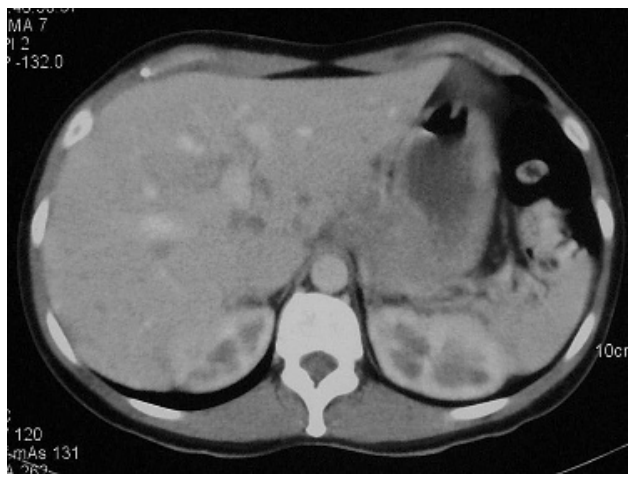

Figure 1 CT scan of the abdomen.

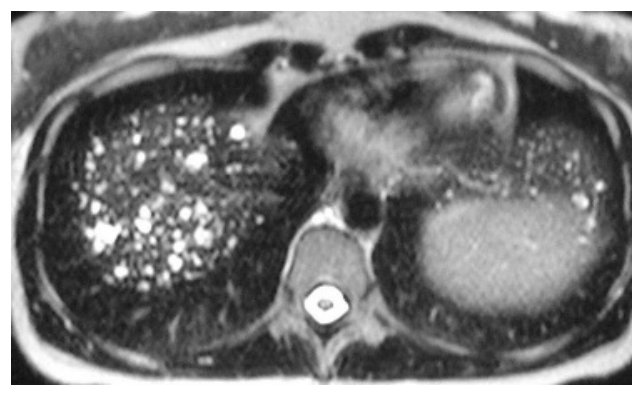

Figure 2 Axial T2-weighted MRI scan of the abdomen.
Robin Spiller, Editor

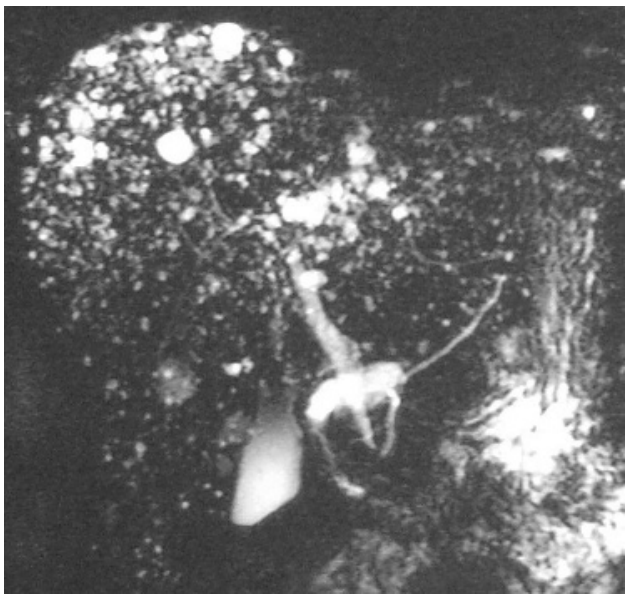

Figure 3 Coronal T2-weighted MRI scan of the liver.

abdominal ultrasound identified an enlarged liver with coarse echo texture and several focal abnormalities throughout both lobes. A CT scan of the abdomen showed multiple indeterminate areas of low attenuation within the liver (figure 1). MRI of the liver (figures 2,3) provided further characterisation.

\section{Question}

What is the diagnosis and what further investigation and follow-up is needed?

See page 1352 for answer

This case is submitted by:

J Sharkey, K R Palmer, I D R Arnott Gastrointestinal Unit, Western General Hospital, Edinburgh, UK S Glancy Department of Radiology, Western General Hospital, Edinburgh, UK

Correspondence to: lan D R Arnott, Gastrointestinal Unit, Western General Hospital, Crewe Road, Edinburgh EH4 2XU, UK; ian.arnott@luht.scot.nhs.uk doi: $10.1136 /$ gut.2006.104893 